Vietnam Journal of Mechanics, VAST, Vol. 42, No. 4 (2020), pp. 375-383

DOI: https://doi.org/10.15625/0866-7136/14875

\title{
SIZE DEPENDENT OF THE EFFECTIVE CONDUCTIVITY OF COMPOSITE WITH IMPERFECT INTERFACES
}

\author{
Nguyen Trung Kien ${ }^{1, *}$ \\ ${ }^{1}$ Research and Application Center for Technology in Civil Engineering, \\ University of Transport and Communications, Hanoi, Vietnam \\ *E-mail: ntkien@utc.edu.vn
}

Received: 08 March 2020 / Published online: 05 May 2020

\begin{abstract}
Based on the circle assemblage model, the effective properties of the inclusion with imperfect interface are derived. The equivalent inclusion is incorporated in the Fourier Transform algorithm to determine the effective conductivity of the composite with lowly conducting or highly conducting interface. The size effect is considered for both cases. Numerical results are provided to illustrate the dependence of the effective conductivity on the size of inhomogeneities.
\end{abstract}

Keywords: effective conductivity, imperfect interface, size effect.

\section{INTRODUCTION}

Many studies concerning the effective property of composite assume a perfect interface between the constituent phases. However, due to presence of roughness or mismatch between the phases, this assumption is not appropriate. In the context of thermal conduction, two kinds of imperfect interface models are considered. The first one is the well-known Kapitza's thermal resistance model [1] called also lowly conducting interface model (LC), according to which the temperature is discontinuous across an interface and the normal heat flux component is continuous and proportional to the temperature jump across the interface. Dual to the first model, the highly conducting interface model (HC) assumes that the normal heat flux is discontinuous across the interface while the temperature is continuous across it. The theoretical studies of this problem were conducted by Benveniste and Miloh [2,3], Hasselman and Johnson [4], Torquato and Rintoul [5], Hashin [6], Le et al. [7]. The lowly conducting interface model or the highly conducting interface model can be derived in an asymptotic way from the physically-based configuration where a very thin lowly or highly conducting interphase is situated between two constituent phases. Numerical modelling of the effective conductivity of composite with imperfect interface were proposed by Le et al. [7,8], Monchiet [9]. An important point to all these works is that the effective conductivity of the composites depends on the size 
of the inhomogeneity. The objective of the present paper is to investigate the size dependence of the effective conductivity of composite media with imperfect interfaces in two-dimensional space. The paper is structured as follows. Section 2 presents a model of inclusion with imperfect interface. In Section 3, the equivalent inclusion incorporating with the Fourier Transform algorithm is presented. In Section 4, the size effect on the effective conductivity of composite is numerically illustrated. A few remarks are provided in the last part.

\section{INCLUSION WITH IMPERFECT INTERFACE}

Let us consider a representative volume element $V$ of Hashin-Shtrikman two-phase coated circle assemblage. Here, circle of phase 1 are coated by circular shells of phase 2. The relative volume proportions and coating orders of the phases in all compound circles are the same. The space of $V$ is entirely filled by such compound circles distributed randomly with diameters varying to infinitely small. In this case, $v_{1}, c_{1}$ are the volume fraction and conductivity of the inclusion phase, while $v_{2}, c_{2}$ are referred to as the volume fraction and conductivity of the matrix phase. With the thin coating $\left(v_{2} \ll 1\right)$, one obtains asymptotically expression of the effective conductivity of isotropic materials $[10,11]$

$$
c^{e f f}=c_{1}+v_{2} \frac{\left(c_{2}-c_{1}\right)\left(c_{1}+c_{2}\right)}{2 c_{2}}+O\left(v_{2}^{2}\right) .
$$

In the case of thin coating thickness $h\left(\frac{h}{R_{1}} \ll 1, R_{1}\right.$ is the radii of inner circle) one has

$$
\frac{1}{v_{1}}=\frac{1}{1-v_{2}}=1+v_{2}+O\left(v_{2}^{2}\right)
$$

further

$$
\frac{1}{v_{1}}=\frac{R_{2}^{2}}{R_{1}^{2}}=\left(1+\frac{h}{R_{1}}\right)^{2}=1+\frac{2 h}{R_{1}}+O\left(\frac{h^{2}}{R_{1}^{2}}\right),
$$

hence

$$
v_{2}=\frac{2 h}{R_{1}}+O\left(\frac{h^{2}}{R_{1}^{2}}\right)
$$

\subsection{Inclusion with lowly conducting interface}

In the lowly conducting imperfect interface model [7], it is assumed that

$$
c_{2}=\frac{h}{\alpha_{K}}, \quad \frac{h}{R_{1}} \rightarrow 0,
$$

where $\alpha_{K}$ is called the Kapitza thermal resistance.

Substituting (4) and (5) into (1), one derives the asymptotic expression of the effective conductivity $c^{1 L}$ of the assemblage of circular inclusions of phase 1 coated by the infinitely thin shell of thermal resistance $\alpha_{K}$

$$
c^{1 L}=\frac{c_{1}}{1+\frac{c_{1} \alpha_{K}}{R_{1}}}+O\left(\frac{h}{R_{1}}\right) .
$$




\subsection{Inclusion with highly conducting interface}

In the highly conducting imperfect interface model [7], it is assumed that

$$
c_{2}=\frac{c_{s}}{h}, \quad \frac{h}{R_{1}} \rightarrow 0,
$$

where $c_{S}$ is called the surface conductivity.

Substituting (4) and (7) into (1), one derives the asymptotic expression of the effective conductivity $c^{1 H}$ of the assemblage of circular inclusions of phase 1 coated by the infinitely thin shell of surface conductivity $c_{s}$

$$
c^{1 H}=c_{1}+\frac{2 c_{s}}{R_{1}}+O\left(\frac{h}{R_{1}}\right) .
$$

\section{FFT SIMULATION FOR COMPOSITE WITH IMPERFECT INTERFACE}

Consider a periodic composite reinforced by fibers aligned in the direction $\mathrm{Ox}_{3}$. The fibers are randomly distributed in the unit cell. The contact between fibers and matrix is the imperfect interface. This problem can be modelled as a composite of three-component (see for instance [6,7]): circular inclusion $\left(v_{1}, c_{1}\right)$, matrix $\left(v_{M}, c_{M}\right)$ and interphase with volume fraction $v_{2}=\frac{2 h}{R_{1}}\left(R_{1}\right.$ is radii of the inner circle), conductivity $c_{2}=\frac{h}{\alpha_{K}}$ or $c_{2}=\frac{c_{s}}{h}$ (Fig. 1(a)). By substitution scheme, one can consider the two-component composite with perfect interface (Fig. 1(b)) in which the conductivity of equivalent inclusion is determined by (6) or (8)

$$
\begin{aligned}
c_{E I} & =c^{1 L}=\frac{c_{1}}{1+\frac{c_{1} \alpha_{K}}{R_{1}}} \quad \text { for lowly conducting } \\
\text { or } \quad c_{E I} & =c^{1 H}=c_{1}+\frac{2 c_{s}}{R_{1}} \quad \text { for highly conducting }
\end{aligned}
$$

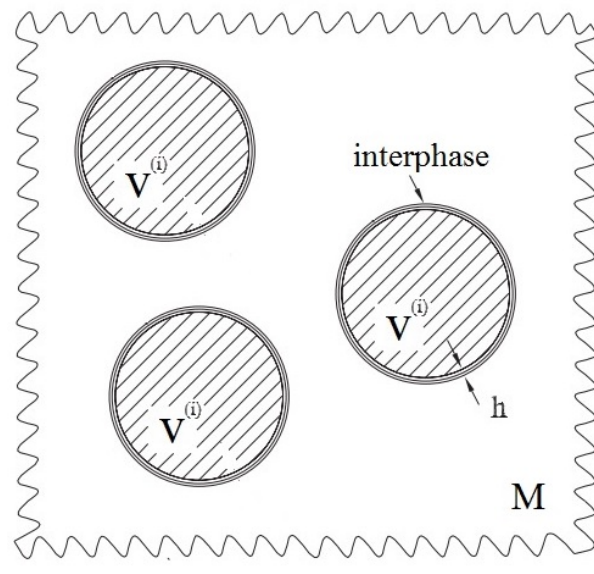

(a)

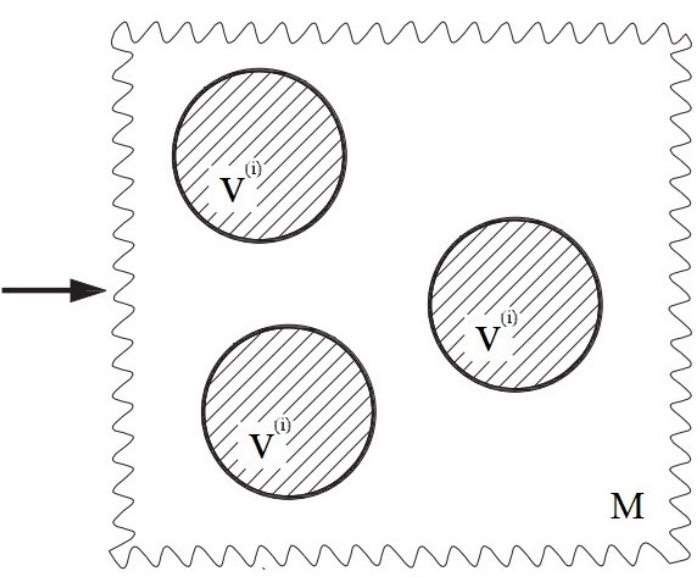

(b)

Fig. 1. Unit cell: coated circle assemblage randomly distributed (a), equivalent inclusion (b) 
The algorithm for determining the effective conductivity of two-phase periodic materials based on the Fourier transform method (FFT) has been introduced in the literature [12]. In the next section, this method will be applied to calculate the effective conductivity of the composite with imperfect interface.

\section{SIZE EFFECT ON THE EFFECTIVE CONDUCTIVITY}

In this section, we will study the size dependent of the effective conductivity of the fiber-matrix composites. A unit cell having the dimension 1 along each space directions is considered containing $N_{\text {inclu }}$ circular inclusions non overlapping with the same radius $R_{1}$ (Fig. 2).
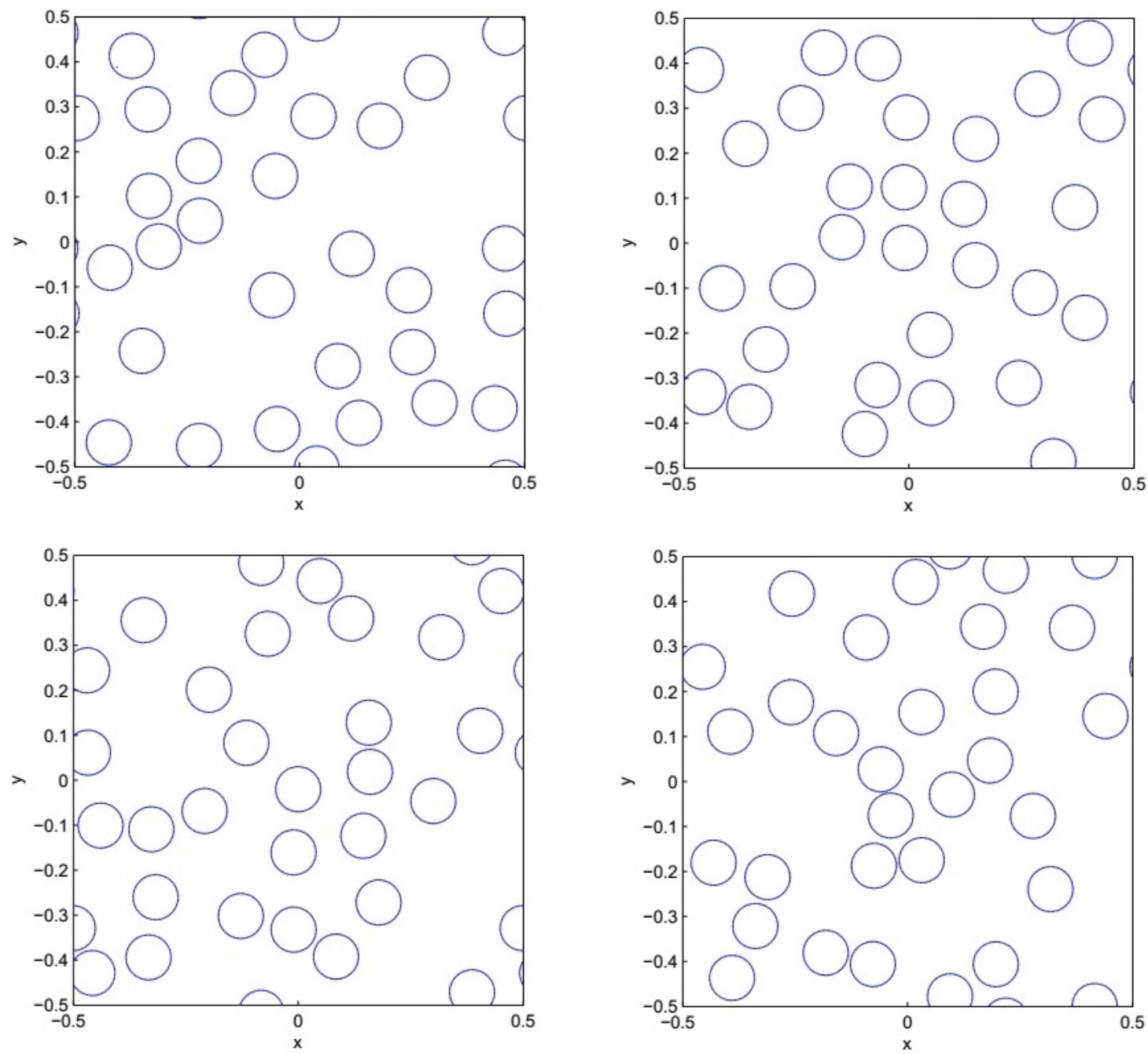

Fig. 2. Unit cell with randomly distributed inclusions 


\subsection{Lowly conducting interface}

To investigate the size effect on the effective properties of the composites, one introduces the dimensionless Kapitza resistance

$$
\alpha_{K}^{*}=\frac{\alpha_{K} c_{M} R_{1}^{*}}{R_{1}}
$$

with $R_{1}^{*}$ is the dimensionless radius

$$
R_{1}^{*}=\frac{R_{1}}{L} .
$$

In practice, the size dependent of the effective conductivity is studied by increasing (decreasing) the radius of the inclusions $R_{1}$ or by decreasing (increasing) the dimensionless Kapitza resistance $\alpha_{K}^{*}$ while the dimensionless radius $R_{1}^{*}$ is kept constant. Consider the unit cell containing $N_{\text {inlcu }}=30$ inclusions. The volume fraction of reinforcement is $N_{\text {inlcu }} \pi R_{1}^{2} / L^{2}=0.236$. The thermal conductivities of the matrix and the inclusion are $c_{M}=1 \mathrm{Wm}^{-1} \mathrm{~K}^{-1}, c_{1}=10 \mathrm{Wm}^{-1} \mathrm{~K}^{-1}$ while the thermal resistance of the interface is $\alpha_{K}=10^{-5} \mathrm{~m}^{2} \mathrm{~K} / \mathrm{W}$. In this example, $R_{1}^{*}=0.05$ and the radius $R_{1}$ vary from $0.02 \mu \mathrm{m}$ to $250 \mu \mathrm{m}$. The effective conductivity is obtained from algorithm of FFT in [12] in which the conductivity of the equivalent inclusion is determined from first equation of (9). The values of the effective conductivity $C_{11}^{e f f}$ and $C_{22}^{\text {eff }}$ for each occurrences are respectively presented on Figs. 3, 4 for $R_{1}=50 \mu \mathrm{m}$. The cumulated average value of the effective conductivity is also computed.

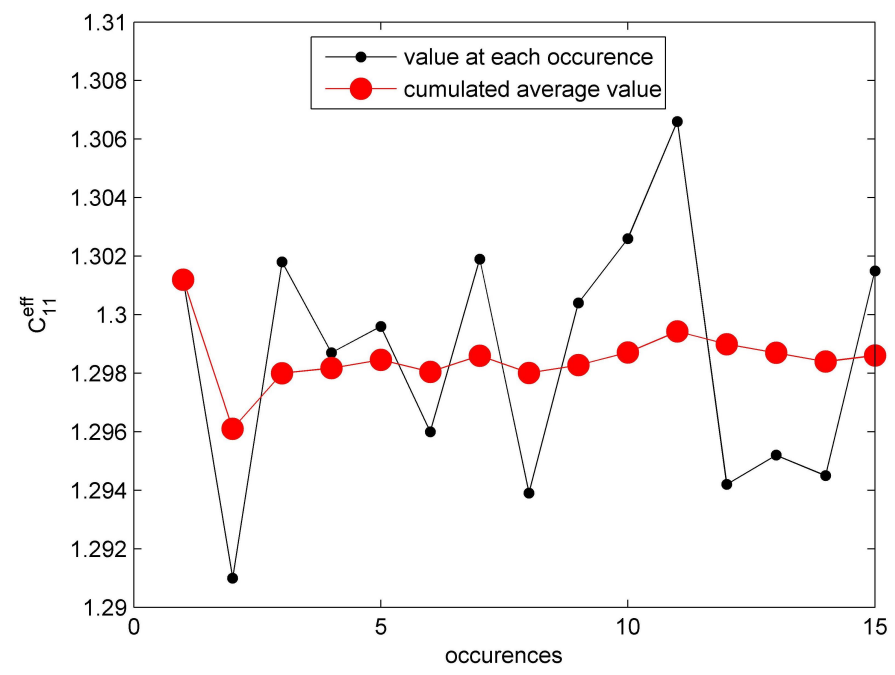

Fig. 3. Statistical convergence of the effective conductivity $C_{11}^{e f f}$ for $R=50 \mu \mathrm{m}$

On Tab. 1, we present the cumulated average value of the effective conductivity $C_{11}^{e f f}$ and $C_{22}^{e f f}$ for each inclusion radius. A small difference between the results related to both directions but the behavior is also practically isotropic. The Mori-Tanaka scheme and the 


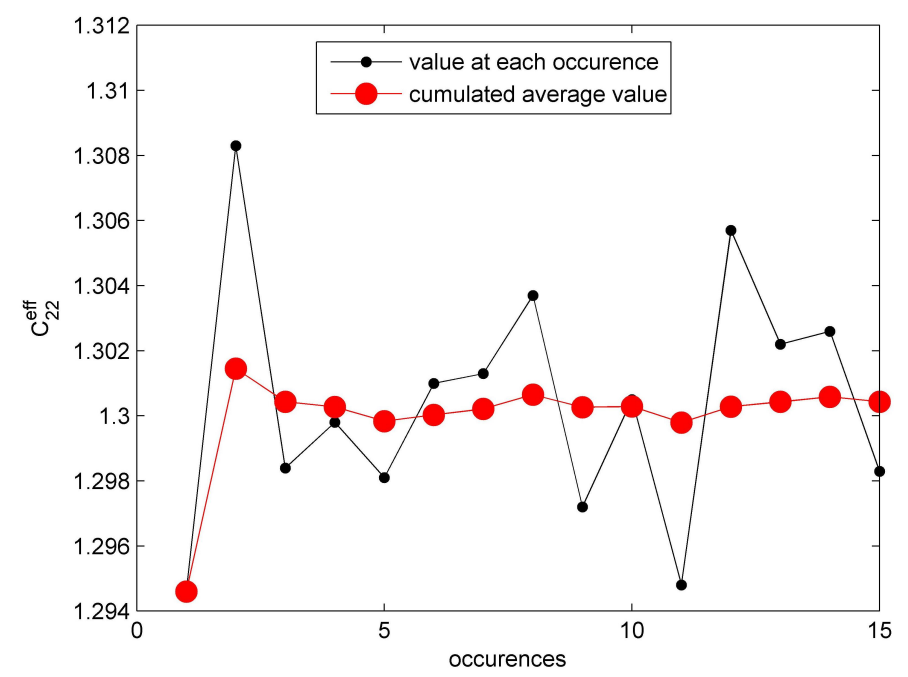

Fig. 4. Statistical convergence of the effective conductivity $C_{22}^{e f f}$ for $R=50 \mu \mathrm{m}$

Table 1. Cumulated average value of the effective conductivities obtained with 15 occurrences. Case of lowly conducting imperfect interface

\begin{tabular}{cccccccc}
\hline$R_{1}$ (in $\left.\mu \mathrm{m}\right)$ & 0.02 & 0.1 & 0.5 & 2.5 & 12.5 & 50 & 250 \\
\hline$C_{11}^{\text {eff }}$ & 0.6048 & 0.6105 & 0.6375 & 0.7466 & 1.0252 & 1.2986 & 1.4587 \\
$C_{22}^{\text {eff }}$ & 0.6081 & 0.6136 & 0.6402 & 0.7479 & 1.0252 & 1.3004 & 1.4631 \\
Monchiet [9] & 0.6289 & 0.6336 & 0.6565 & 0.7541 & 1.0252 & 1.2993 & 1.4526 \\
Mori-Tanaka & 0.6199 & 0.6248 & 0.6484 & 0.7494 & 1.0251 & 1.2906 & 1.4323 \\
\hline
\end{tabular}

numerical results of Monchiet presented in [9] are also included for comparison. It can be seen that the results of present approach are slightly different to those of Monchiet. In [9], the discontinuities of the temperature are simulated by adding a temperature field of third order polynomial which are null outside of the inclusion but are different of zero inside the inclusion. This method gives accurate results but requires more time calculations and computer memory. With the equivalent-inclusion approach, the FFT simulation in this paper provides a simpler method for computing the effective conductivity while still ensuring accuracy.

\subsection{Highly conducting interface}

Similarly to the previous section, one introduces the dimensionless surface conductivity

$$
c_{s}^{*}=\frac{c_{s} R_{1}^{*}}{c_{M} R_{1}}
$$


Consider the same micro-structure as previous section, the volume fraction of reinforcement is 0.236 with 30 inclusions. The inclusion is assumed to be less conducting than the matrix one. The thermal conductivities of the matrix and the inclusion are $c_{M}=$ $1 \mathrm{Wm}^{-1} \mathrm{~K}^{-1}, c_{1}=0.1 \mathrm{Wm}^{-1} \mathrm{~K}^{-1}$ while the surface conductivity is $c_{s}=10^{-6} \mathrm{~W} / \mathrm{K}$. The radius $R_{1}$ vary from $0.02 \mu \mathrm{m}$ to $250 \mu \mathrm{m}$. The effective conductivity is obtained from algorithm of FFT in [12] in which the conductivity of the equivalent inclusion is determined from second equation of (9). The cumulated average value of the effective conductivity $C_{11}^{e f f}$ and $C_{22}^{e f f}$ are presented in Tab. 2.

Table 2. Cumulated average value of the effective conductivities obtained with 15 occurrences. Case of highly conducting imperfect interface

\begin{tabular}{cccccccc}
\hline$R_{1}$ (in $\left.\mu \mathrm{m}\right)$ & 0.02 & 0.1 & 0.5 & 2.5 & 12.5 & 50 & 250 \\
\hline$C_{11}^{e f f}$ & 1.6714 & 1.5867 & 1.3465 & 0.9755 & 0.7545 & 0.6924 & 0.6739 \\
$C_{22}^{\text {eff }}$ & 1.6797 & 1.5930 & 1.3487 & 0.9755 & 0.7555 & 0.6941 & 0.6759 \\
Mori-Tanaka & 1.6006 & 1.5422 & 1.3343 & 0.9755 & 0.7569 & 0.6982 & 0.6811 \\
\hline
\end{tabular}

The variations of the effective conductivity with the size of the inclusions are presented on Figs. 5 and 6 . The size dependent with the inclusion radius is clearly observed.

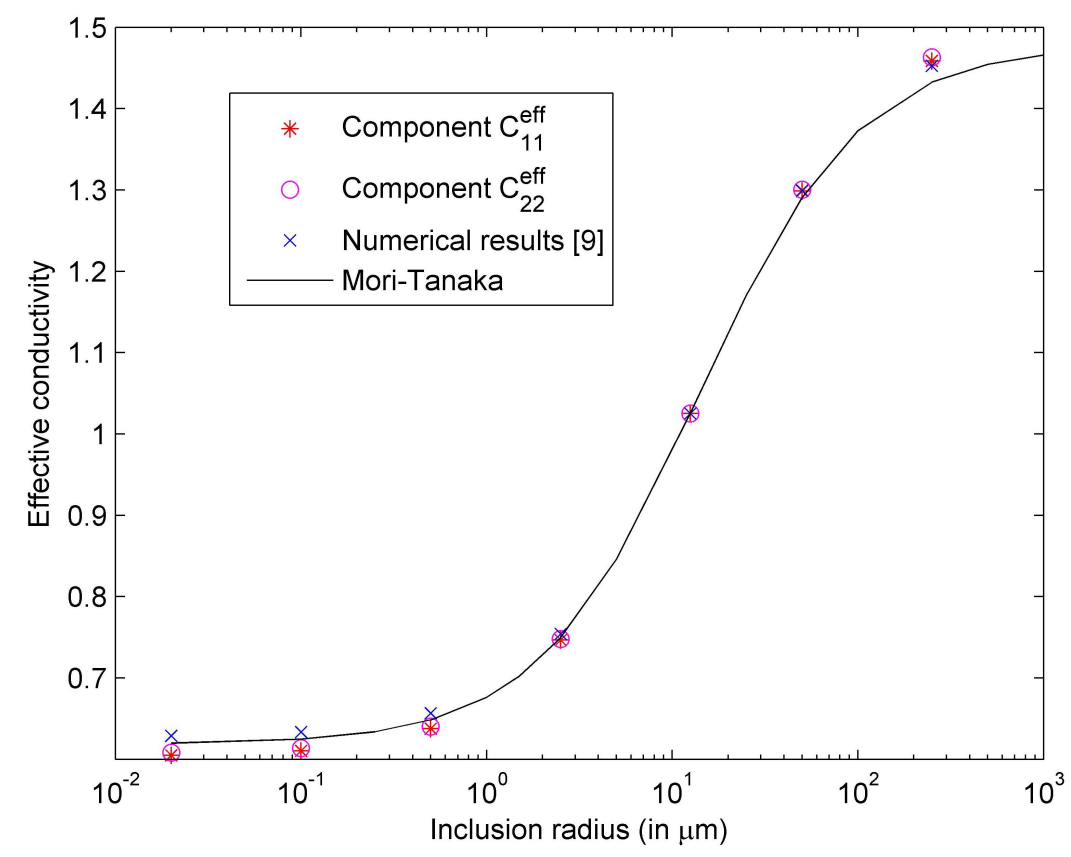

Fig. 5. Variations of the effective conductivity with the radius of inclusion for the case of lowly conducting interface 


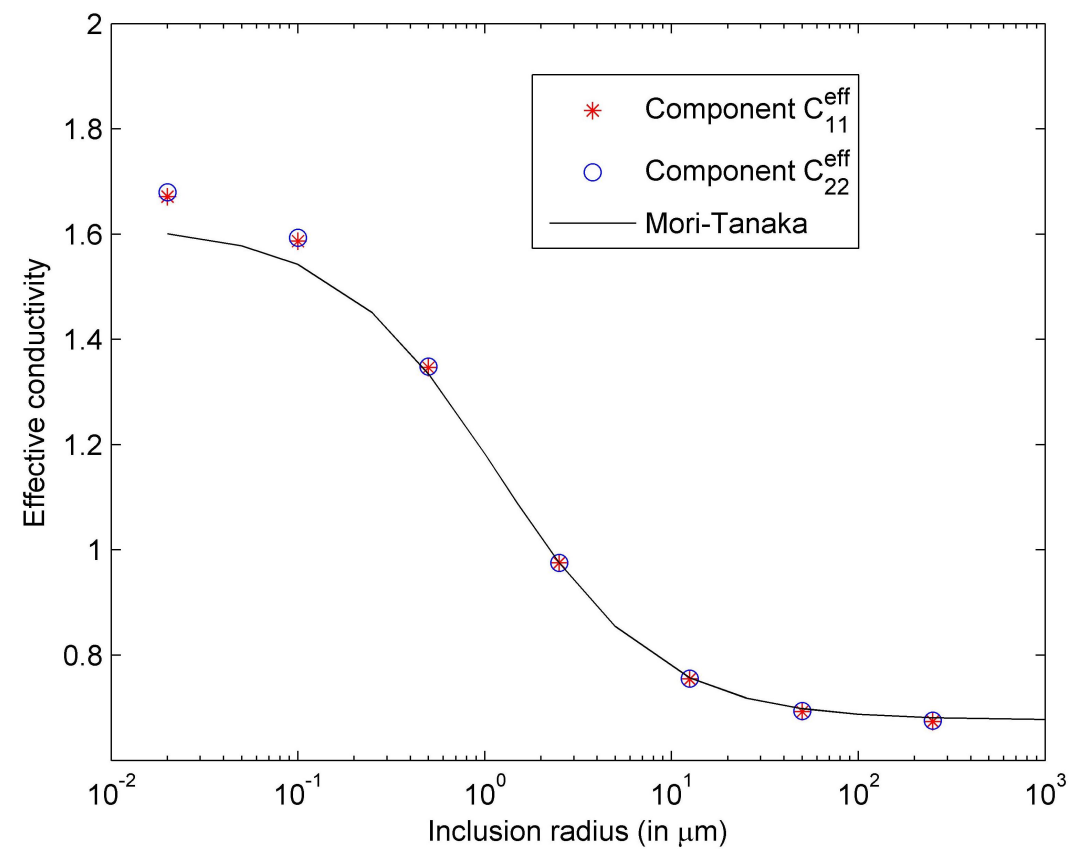

Fig. 6. Variations of the effective conductivity with the radius of inclusion for the case of highly conducting interface

\section{CONCLUSION}

In this paper, the plane problem of periodic composite containing random distributed fibres was studied. The effect of the imperfect interface is taken into account. The principle of the approach is to consider a very thin lowly or highly conducting interphase situated between the fibers and matrix. The inclusion and thin interphase are considered as a coated-inclusion and can be replaced by the equivalent inclusion whose conductivity depends on the radius, the conductivity of the inclusion and the conductivity of the interphase (9). The equivalent inclusion is incorporated in the Fourier Transform algorithm to compute the effective conductivity of composites with lowly conducting (LC) or highly conducting $(\mathrm{HC})$ interfaces. The results show that the effect of LC imperfect interfaces decrease the effective conductivity of the composite when the heterogeneity size becomes small. Inversely, the effect of HC imperfect interfaces increase the effective conductivity of the composite when the radius of inclusion decrease. The comparison with other numerical results and analytic solutions coming from the Mori-Tanaka scheme show good agreement. The method could be extend to other physical problems involving surface discontinuities.

\section{ACKNOWLEDGMENT}

This research is supported by Vietnam National Foundation for Science and Technology Development (NAFOSTED) under grant number 107.02-2019.13 


\section{REFERENCES}

[1] P. L. Kapitza. The study of heat transfer in helium II. J. Phys. (USSR), 4, (1941), pp. 181-210.

[2] Y. Benveniste and T. Miloh. The effective conductivity of composites with imperfect thermal contact at constituent interfaces. International Journal of Engineering Science, 24, (9), (1986), pp. 1537-1552. https://doi.org/10.1016/0020-7225(86)90162-x.

[3] T. Miloh and Y. Benveniste. On the effective conductivity of composites with ellipsoidal inhomogeneities and highly conducting interfaces. Proceedings of the Royal Society of London. Series A: Mathematical, Physical and Engineering Sciences, 455, (1987), (1999), pp. 2687-2706. https://doi.org/10.1098/rspa.1999.0422.

[4] D. P. H. Hasselman and L. F. Johnson. Effective thermal conductivity of composites with interfacial thermal barrier resistance. Journal of Composite Materials, 21, (6), (1987), pp. 508515. https://doi.org/10.1177/002199838702100602.

[5] S. Torquato and M. D. Rintoul. Effect of the interface on the properties of composite media. Physical Review Letters, 75, (22), (1995). https://doi.org/10.1103/physrevlett.76.3241.

[6] Z. Hashin. Thin interphase/imperfect interface in conduction. Journal of Applied Physics, 89, (4), (2001), pp. 2261-2267. https://doi.org/10.1063/1.1337936.

[7] Q. H. Le, D. C. Pham, G. Bonnet, and Q.-C. He. Estimations of the effective conductivity of anisotropic multiphase composites with imperfect interfaces. International Journal of Heat and Mass Transfer, 58, (1-2), (2013), pp. 175-187. https://doi.org/10.1016/j.ijheatmasstransfer.2012.11.028.

[8] Q. H. Le, T. L. Phan, and G. Bonnet. Effective thermal conductivity of periodic composites with highly conducting imperfect interfaces. International Journal of Thermal Sciences, 50, (8), (2011), pp. 1428-1444. https://doi.org/10.1016/j.ijthermalsci.2011.03.009.

[9] V. Monchiet. FFT based iterative schemes for composites conductors with non-overlapping fibers and Kapitza interface resistance. International Journal of Solids and Structures, 135, (2018), pp. 14-25. https://doi.org/10.1016/j.ijsolstr.2017.10.015.

[10] D. C. Pham. Solutions for the conductivity of multi-coated spheres and spherically symmetric inclusion problems. Zeitschrift für angewandte Mathematik und Physik, 69, (1), (2018). https://doi.org/10.1007/s00033-017-0905-6.

[11] T. K. Nguyen, V. L. Nguyen, and D. C. Pham. Effective conductivity of isotropic composite with Kapitza thermal resistance. Vietnam Journal of Mechanics, 40, (4), (2018), pp. 377-385. https://doi.org/10.15625/0866-7136/12936.

[12] V. L. Nguyen and T. K. Nguyen. FFT simulations and multi-coated inclusion model for macroscopic conductivity of 2D suspensions of compound inclusions. Vietnam Journal of Mechanics, 37, (3), (2015), pp. 169-176. https://doi.org/10.15625/0866-7136/37/3/5096. 